\title{
Tracking Source azimuth Using a Single Vector Sensor
}

\author{
Paulo Felisberto, Paulo Santos, Sérgio M. Jesus \\ Institute for Systems and Robotics \\ Universidade do Algarve \\ 8005-139 Faro, Portugal \\ \{pfelis,pjsantos, sjesus\}@ualg.pt
}

\begin{abstract}
This paper aims at estimating the azimuth of an underwater acoustic source with a single vector sensor. A vector sensor is a device that measures the scalar acoustic pressure field and the vectorial acoustic velocity field at a single location in space. The actual sensor technology allows to build compact vector sensors, with an operational frequency response ranging from a few hertz to several tens of kilohertz, thus the same device can be used to receive shipping noise upto dolphin whistles. It is demonstrated that one can attain a reliable estimate of the azimuth of a source with a single vector sensor. The method presented is based on the inner product between the sampled acoustic field and the different particle velocity orthogonal components. The method is very simple and low computational demanding thus, well suited to be used in mobile or light platforms where space and/or computational power requirements is of concern. It is shown that the proposed method can be used either in time or in frequency domain, giving rise to easily estimating the azimuth of several sources with non-overlapping frequency bands. The data discussed herein (ship noise, communication signals, tomographic signals) were acquired during the Makai'2005 experiment using a four element vector sensor array. It is shown that the estimates obtained with a single vector sensor are comparable with those obtained with the full vector sensor array and are inline with the expected results as known from the geometry of the experiment.
\end{abstract}

Keywords-azimuth estimation, underwater acoustic vector sensor, sonar signal processing.

\section{INTRODUCTION}

An acoustic vector sensor is a device that measures the three orthogonal components of the particle velocity, simultaneously with the pressure field at a single position in space. Vector sensors have been used for a long time by the US Navy in SONAR and target location due to their inherent spatial filtering capabilities [1]. In the early nineties, a paper by D'Spain et al. [2], presented results for single-element and full array beamforming data acquired by an array of 16 vector sensors, the DIFAR array. This paper received considerable attention, and during the last two decades several authors have conducted research on the signal processing theory of vector sensors ( [3] and references therein). Although, the majority of those works are related to direction of arrival estimation and source localization recently, vector sensors have been proposed in other fields like port and waterway security [4], underwater communications [5] and geoacoustic inversion [6].
Vector sensors have been developed using different technological principles [7]. Recently, thanks to technological progress small size, low noise vector sensors with improved characteristics (dynamic range, bandwidth) became available [8]. Those compact sensors are well suited to be used in light systems, where space is limited. Example of such systems are AUV and similar mobile platforms.

This paper shows that using such a device, Wilcoxin TV001 [9], in a shallow water environment, one can track the azimuth of slowly moving high frequency sources in a range upto $2 \mathrm{~km}$. It is also shown that with the same device the orientation of the low frequency ship noise can be determined. The method used is an intensity-based algorithm [2] [10], that can be used for broadband signals (time domain) or narrowband signals (frequency domain). This method demands low computational power, becoming suitable to be used in light/mobile nodes in systems with distributed intelligence [7].

The single vector sensor azimuth estimates for 4 vector sensors considered individually were compared with estimates obtained by applying an array beamforming technique (same sensors) presented in [11]. The results show that the performance of the single vector method is equivalent to that of array beamforming technique.

This paper is organized as follows: the theoretical framework considered in data processing and analysis is depicted in Section II; Section III presents the experimental setup and a discussion of the results and Section IV summarizes the paper.

\section{THEORETICAL FRAMEWORK}

This section presents the measurement model for a single vector sensor and the intensity based method to estimate source azimuth. A time domain estimator and this frequency domain counterpart are derived.

\section{A. Measurement model}

In the following, a vector sensor that measures the pressure $p(t)$ and the three orthogonal components of the particle velocity along $x\left(v_{x}(t)\right), y\left(v_{y}(t)\right)$ and $z$-axis $\left(v_{z}(t)\right)$ in a point of space is considered. The vector sensor is positioned at the origin of the Cartesian system, being the $x y$-plane the horizontal plane and $x z$-plane the vertical 
plane. The azimuth $\Theta(-180 \leq \Theta \leq 180)$ and elevation $\Phi(-90 \leq \Phi \leq 90)$ angles are defined in a conventional manner.

Without loss of generality it is assumed that the signal impinging the vector sensor is in the far-field and is band limited, thus pressure and frequency are related by the linearized acoustic equation [10], by

$$
-\nabla p=\rho_{0} \frac{\partial \mathbf{v}}{\partial t}
$$

where $\rho_{0}$ is the water density and $\mathbf{v}=\left[v_{x}, v_{y}, v_{z}\right]$ is the particle velocity vector.

For a narrowband signal at frequency $\omega_{0},(1)$ can be written as [5]

$$
\begin{aligned}
& v_{x}(t)=-\frac{1}{j \rho_{0} \omega_{0}} \frac{\partial p(t)}{\partial x}, \\
& v_{y}(t)=-\frac{1}{j \rho_{0} \omega_{0}} \frac{\partial p(t)}{\partial y}, \\
& v_{z}(t)=-\frac{1}{j \rho_{0} \omega_{0}} \frac{\partial p(t)}{\partial z} .
\end{aligned}
$$

The above equations state that the particle velocity in a given direction is proportional to the pressure derivative in that direction, but with an opposite sign. Thus assuming that $s(t)$ is the pressure at the vector sensor due to a point source located at azimuth $\Theta_{s}$ and elevation $\Phi_{s}$, with space-time evolution given by $\left.e^{j \omega_{0}(t+\mathbf{r} . \mathbf{u} / c}\right)$ (plane-wave assumption), where $\mathbf{r}$ is the positioning vector of the source, $\mathbf{u}=$ $\left[u_{x}, u_{y}, u_{y}\right]=\left[\cos \left(\Phi_{s}\right) \cos \left(\Theta_{s}\right), \cos \left(\Phi_{s}\right) \sin \left(\Theta_{s}\right), \sin \left(\Phi_{s}\right)\right]$ represents its direction cosines, and $c$ is the sound speed in the water, the following system of equations represent the measurement model of a vector sensor,

$$
\begin{aligned}
p(t) & =s(t)+n(t), \\
v_{x}(t) & =-A u_{x} s(t)+n_{x}(t), \\
v_{y}(t) & =-A u_{y} s(t)+n_{y}(t), \\
v_{z}(t) & =-A u_{z} s(t)+n_{z}(t) .
\end{aligned}
$$

The proportionality factor $A$ arises directly from (2), but in a more general approach can also account for any existing proportionality in the output stream of a vector sensor device due to different electro-mechanical principles used to measure pressure and particle velocity. In (3) $n(t)$ represents additive noise pressure, and $n_{x}(t), n_{y}(t), n_{z}(t)$ its particle velocity counterparts. A common assumption is that signal and noise are uncorrelated both in time and space. The cross-correlation between the 4 components of a vector sensor have been studied by several authors [12] [13]. It was demonstrated that in presence of azimuthally isotropic noise the horizontal particle velocity components and the pressure are mutually uncorrelated. Moreover, if the noise is spherically symmetric the vertical particle velocity term is also uncorrelated with the other noise terms. Also, the noise power at the pressure channel is equal to the sum of noise power at the so called pressure equivalent of particle velocity measurement [13]. The pressure equivalent of the particle velocity measurement, is obtained as the product of the particle velocity measurement by $-\rho_{0} c[10]$.

\section{B. Intensity based azimuth estimation}

Intensity based source direction estimation was considered in D'Spain et al. [2] pioneer work. Later, Nehorai and Paldi [10] revisited the method and analyzed its statistical performance bounds in terms of Cramér-Rao bound and mean square angular error. The method is based in the cross-correlation between the pressure measurements and the different components of the particle velocity, that allows to estimate the factors $A u_{x}, A u_{y}$ and $A u_{y}$ and subsequently the direction of the impinging source. Taking into account that the signal and the noise are zero mean uncorrelated processes, the pressure related equation and the $x$ component of particle velocity in (3), one can write the cross-correlation at lag 0 between these two vector sensor components as

$$
E\left[v_{x}(t) p(t)\right]=-A u_{x} E\left[s^{2}(t)\right]+E\left[n_{x}(t) n(t)\right],
$$

where $E[]$ is the expectation operator, whereas $E\left[s^{2}(t)\right]$ represents the energy of the signal as seen by the vector sensor. The term $E\left[n_{x}(t) n(t)\right]$ represents the cross-correlation (at lag 0 ) between the pressure and the $x$ component of the particle velocity noise. For a number of practical cases, where the noise components are spatially uncorrelated, the cross-correlation between the particle velocity component and pressure noise (at lag 0) are a fraction of the pressure noise power. Thus for high SNR $A u_{x}$ can be estimated directly from cross-correlation (at lag 0 ) between the pressure and $x$ component of particle velocity. Similar analysis holds for the cross-correlation between pressure, $y$ component of the particle velocity.

Being $s(t)$ and noise components stationary processes, a possible estimator for the azimuthal direction of the source signal $\Theta_{s}$ is given by

$$
\hat{\Theta}_{s}^{t}=\arctan \frac{\left\langle v_{y}(t) p(t)\right\rangle}{\left\langle v_{x}(t) p(t)\right\rangle},
$$

where \langle\rangle stands for time averaging. The full 360 degrees are resolved taking into account the sign of the numerator and denominator of (4).

For the elevation, an equivalent method can not be, in general, applied since due to multipath the energy originated from the source impinges the vector in multiple arrivals from different angles.

Estimator 4 can be implemented in the frequency domain. Assuming that $p(t), v_{x}(t), v_{y}(t)$ are narrow band signals centered at frequency $\omega_{0}$, and $P\left(\omega_{0}\right), V_{x}\left(\omega_{0}\right)$ and $V_{y}\left(\omega_{0}\right)$, are their respective frequency bins, one can write

$$
\hat{\Theta}_{s}^{f}=\arctan \frac{\operatorname{real}\left\{V_{y}^{*}\left(\omega_{0}\right) P\left(\omega_{0}\right)\right\}}{\operatorname{real}\left\{V_{x}^{*}\left(\omega_{0}\right) P\left(\omega_{0}\right)\right\}},
$$

where real \{\} represents the real part operator. In case that several narrow band signals are available, their respective 


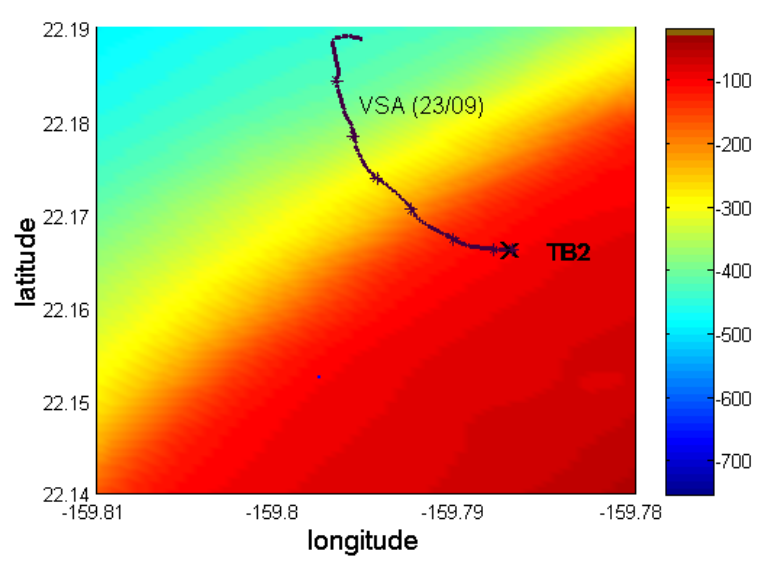

Figure 1. Bathymetry of the experiment. The acoustic source is moored at position represented by a cross and labelled TB2. The black curve represents the ships track during the drift period.

frequency bins should be stacked in (column) vectors $\mathbf{V}_{x}(\omega)$, $\mathbf{V}_{y}(\omega)$ and $\mathbf{P}$ and the estimator becomes

$$
\hat{\Theta}_{s}^{f}=\arctan \frac{\operatorname{real}\left\{\mathbf{V}_{y}^{H}(\omega) \mathbf{P}(\omega)\right\}}{\operatorname{real}\left\{\mathbf{V}_{x}^{H}(\omega) \mathbf{P}(\omega)\right\}},
$$

where $H$ represents the complex conjugate transpose operator. The frequency domain estimator $\hat{\Theta}_{s}^{f}$ above considers only a single signal snapshot, in case that various snapshots are available an average should be used in order to diminish the variance of the estimator.

\section{EXPERIMENTAL RESULTS}

The data set analyzed herein was acquired during the Makai Experiment, that took place off the coast of Kauai I., Hawai, in September 2005.

\section{A. Experimental setup}

The vector sensor acquisition system used in the experiment was composed by 4 sensors, configured in a vertical array with $10 \mathrm{~cm}$ element spacing [9]. The system was suspended off the stern of the research vessel Kilo Moana with a $150 \mathrm{~kg}$ weight at the bottom, to ensure that the array stayed as close to vertical as possible. The $z$-axis was vertically oriented with respect to the bottom, being the deeper sensor at $79.9 \mathrm{~m}$. During the considered period the research vessel drifted 2 and a half hours from a location near a bottom moored acoustic source to a position $2 \mathrm{~km}$ distant. Figure 1 shows the bathymetry of the experimental area with the position of the source, labelled as TB2, and the ship track represented by the black line.

The source was moored $6 \mathrm{~m}$ above the sea floor at $98 \mathrm{~m}$ depth. The emitted signals were sequences of chirps, multitones, M-sequences and communication signals in the $8-14 \mathrm{kHz}$ band. The signal was sampled at $44.1 \mathrm{kHz}$ and recorded in pcm format. Figure 2 presents the spectogram of

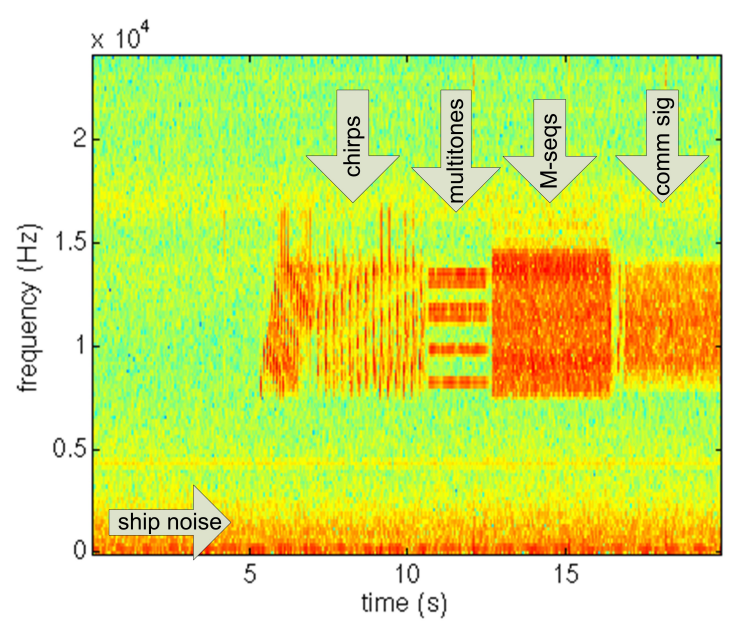

Figure 2. Sample of the signal received in the pressure channel at the deepest vector sensor: a sequence of chirps, multitones, and M-sequences in the $8-14 \mathrm{kHz}$ band from TB2 source, and ship noise in the lower band (bellow $500 \mathrm{~Hz}$ ).

a $20 \mathrm{~s}$ block of the signal acquired by the pressure channel of the deepest vector sensor. In addition to the emitted signals, it can be seen periods of silence in the TB2 band, and ship noise in the lower frequency band.

The received signal was separated in a ship noise band $(90-350 \mathrm{~Hz})$ and in an acoustic source band by linear phase bandpass filtering. The research vessel Kilo Moana noise signature was used to find the orientation of the vector sensor about the $x$-axis, otherwise unknown [11].

\section{B. Azimuth estimation of broadband and narrowband sig-} nals

As discussed in II-B azimuth estimation can be implemented in time domain or in frequency domain. Usually, it is considered that time domain techniques are better suited for broadband processing, whereas frequency domain is for narrowband processing. The signal emitted by the TB2 source is a mixture of waveforms ranging from chirps, a broadband transient signal, to multitones a sum of 8 sinusoids (narrowband signals). In principle in a tracking procedure, one can identify the type of the signal and apply the more convenient method. The tracking procedure can be simplified if the same method can be used for signals of different types. In order to obtain some insight on this subject, next estimations obtained by both methods are compared for the case of a sequence of chirps, and a $2 \mathrm{~s}$ data block of multitones.

1) Chirp sequence: One of the waveforms emitted by TB2 was upsweep LFM chirps lasting $50 \mathrm{~ms}$, followed by $200 \mathrm{~ms}$ of silence in sequences of $4 \mathrm{~s}$.

Figure 3 shows a $1.5 \mathrm{~s}$ long sample of such a sequence as acquired in the pressure channel of a vector sensor, where one can observe several arrivals due to channel multipath. The time domain azimuth estimation using (4) 


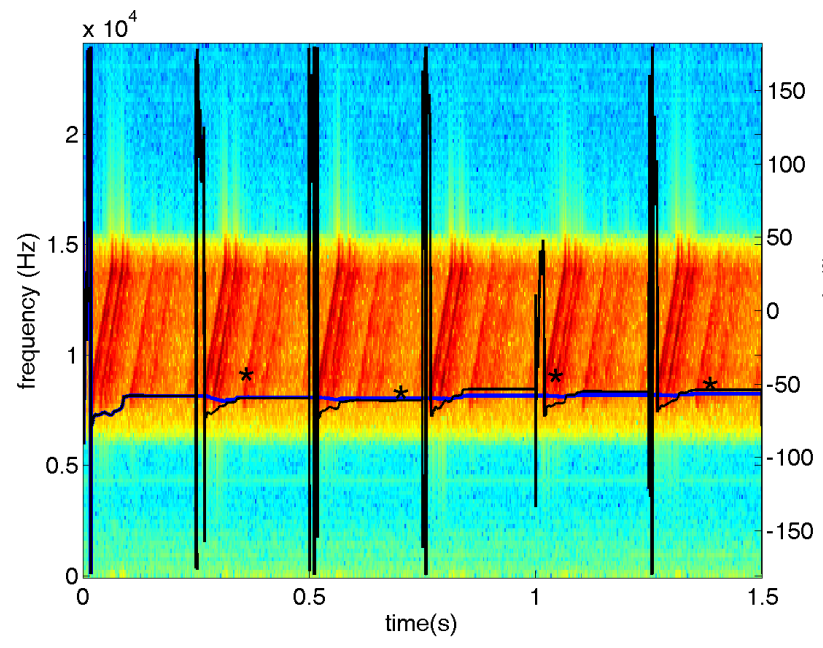

Figure 3. Azimuth estimates of sequence of chirps: iterative estimate in time domain considering the whole sequence (blue line) or each single chirp (black line) and single block frequency domain estimates (asterisks).

was implemented iteratively, thus by plotting the estimates obtained at each iteration, one can get some insight on the convergence rate of the procedure, that is linked with the minimal duration of time averaging that should be considered to attain reliable estimates. The blue line in Figure 3 shows the evolution of the estimates at each instant considering the whole signal from instant 0 , whereas the black lines represents the estimate from the beginning of each chirp. One can observe that it is possible to estimate the azimuth of the source with only one chirp. This suggests that a vector sensor can be used to estimate the azimuth of transient signals when only a single sample of the signal is available.

The frequency domain estimator (6) was applied to the chirp sequence, using non-overlapping blocks of about $340 \mathrm{~ms}$. The block was transformed to frequency domain by a 16384 point FFT, and all the frequency bins in the band of the source where used to estimate its azimuth. The asterisks in Figure 3 presents the estimates obtained at each single block. It can be seen that using such long blocks and the whole available signal band it is possible to obtain reliable estimates without averaging.

2) Multitones: The acoustic source TB2 emitted simultaneous tones at frequencies 8250, 9820, 9914, 11367, 11789, 11882,13078 and $13500 \mathrm{~Hz}$ in blocks of 2 s. Figure 4 shows a spectrogram of such a block.

The azimuth of the source was estimated in the time domain using the same approach as for the chirp sequence. The estimates obtained are represented by the blue line. In the frequency domain it was also used the procedure depicted in III-B1, but two cases were considered: using all tones, represented by asterisks in Figure 4, and using a single frequency $(9820 \mathrm{~Hz})$, represented by a circle. It can

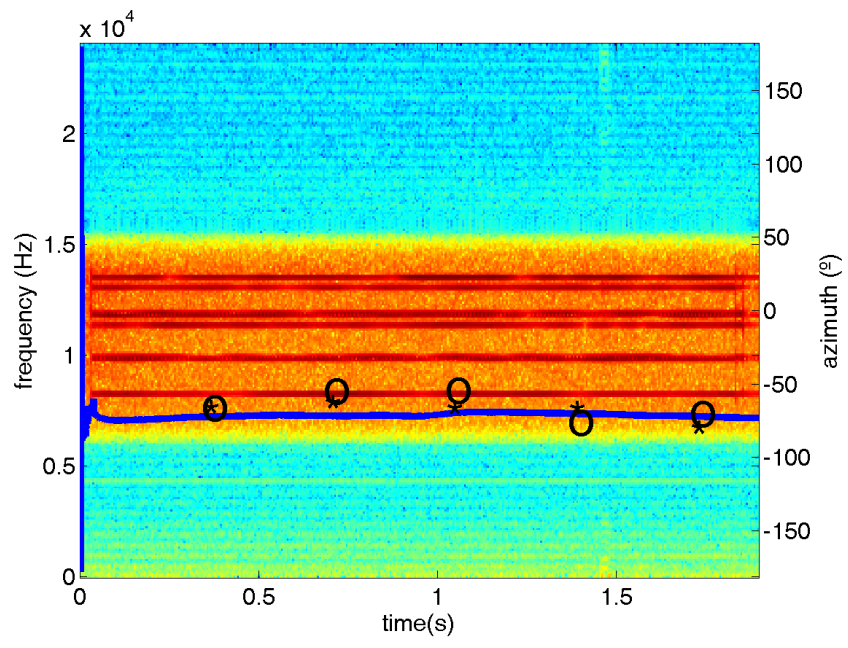

Figure 4. Azimuth estimates of $2 \mathrm{~s}$ lasting multitones composed signal: iterative estimate in time domain considering the $2 \mathrm{~s}$ sample (blue line) and single block frequency domain estimates for a single tone (circles) and all tones (asterisks).

be seen a good agreement between estimates, however one should remark that the estimates obtained with some other tones individually (not shown) suffer of greater variance than for $9820 \mathrm{~Hz}$, thus block averaging should be used.

\section{Azimuth tracking}

Next, it is discussed the results obtained along the 2 and an half hours of ship drifting in the two bands: low frequency ship noise band and high frequency TB2 source band. In both cases the estimates were computed in the frequency domain using the whole band, $90-350 \mathrm{~Hz}$ for ship noise and $8-14 \mathrm{kHz}$ for the TB2 emitted signals. As in III-B1 and III-B2, local estimates were obtained using non-overlapping blocks of $340 \mathrm{~ms}$ signal duration (16384 points FFT). If the signal power in a block was less than $10 \%$ of the average power of all blocks, the estimate was not considered, since it was likely that it corresponds to a period of silence. Although, this procedure was applied to both signal bands, it was only necessary for the source band, since ship noise was continually present. Finally, in order to reduce the number of estimates and their variance, block estimates were used with 25 averages per estimate. Also, the average power of those estimates was computed. The procedure was applied for each vector sensor individually.

Figure 5a) presents the azimuth estimates for each of the 4 vector sensors, and 5b) the average power received by vector sensor 1 (at depth $79.6 \mathrm{~m}$ ), in the ship noise band.

Since the vector sensors were deployed close to the stern of R/V Kilo Moana, with the ship's engine directly above the sensors, this band is dominated by engine noise, with two principal components at frequencies, $180 \mathrm{~Hz}$ and $300 \mathrm{~Hz}$ [11]. The power received by the vector sensors in this band is highly correlated with the power supplied by the engines. 
(a)

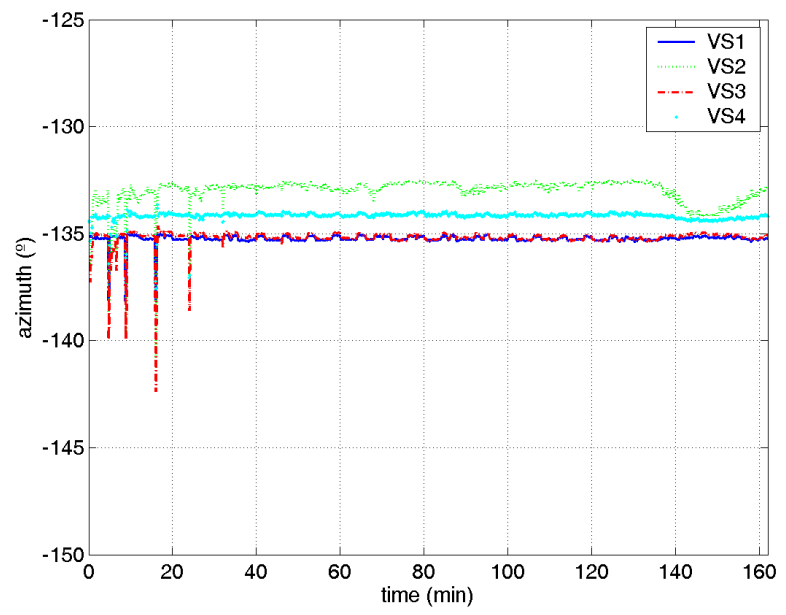

(b)

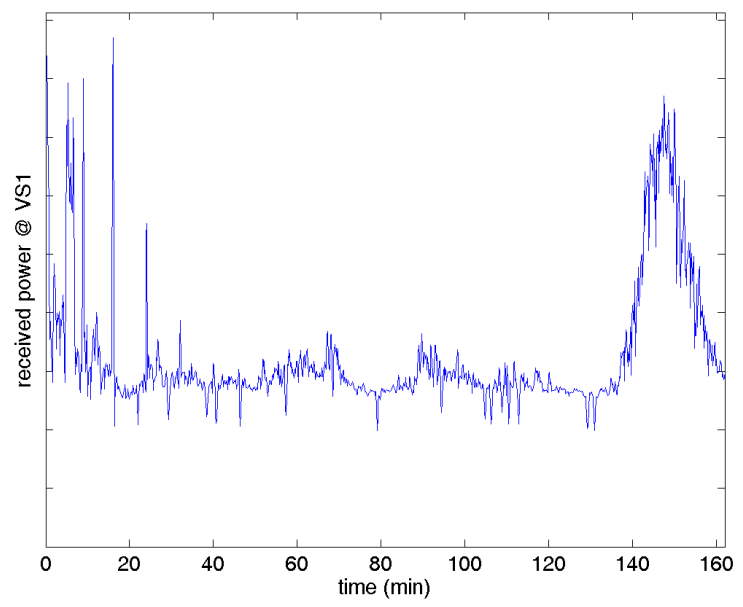

Figure 5. Vector sensor array orientation estimates using ship for each vector sensor during the ship's drifting period a) and ship noise power received at vector sensor $1 \mathrm{~b}$ ).

Observing Figure 5b), it can be seen three distinct periods: an initial period where spike like changes in power occur, presumably due to ships maneuvering; a middle period from minute 30 upto minute 130 when the ship is drifting, the mean power is at a low level with only small changes, and a final period when the power stably increases in a first moment and stably decreases afterwards, most likely due to smooth changes in ship's velocity. The azimuth estimates obtained from the ship noise are highly stable along the 3 periods for all 4 sensors, with small constant differences (less than 2 degrees) between them. However in the first period several instantaneous changes in azimuth estimates occur in all 4 vector sensors, highly correlated with the spikes observed in received power, what suggests important displacements of the vector sensors from their initial vertical (a)

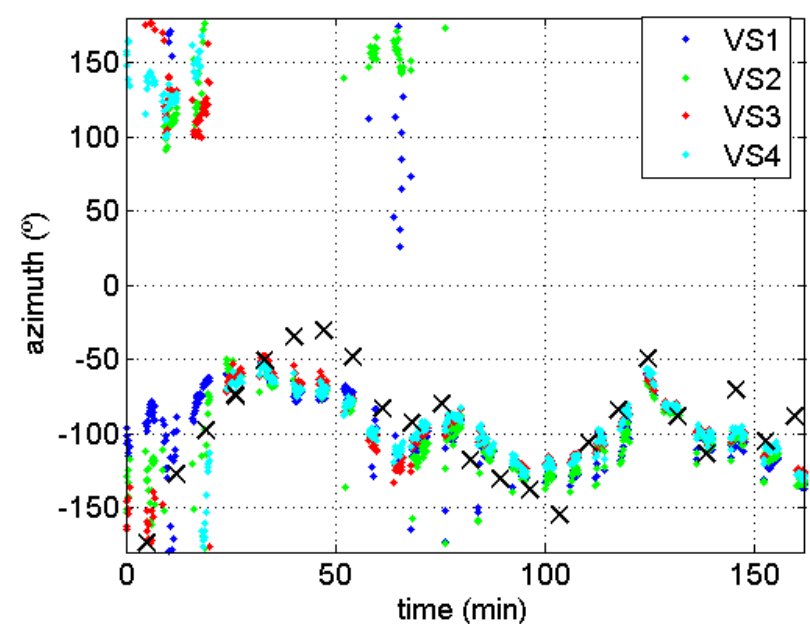

(b)

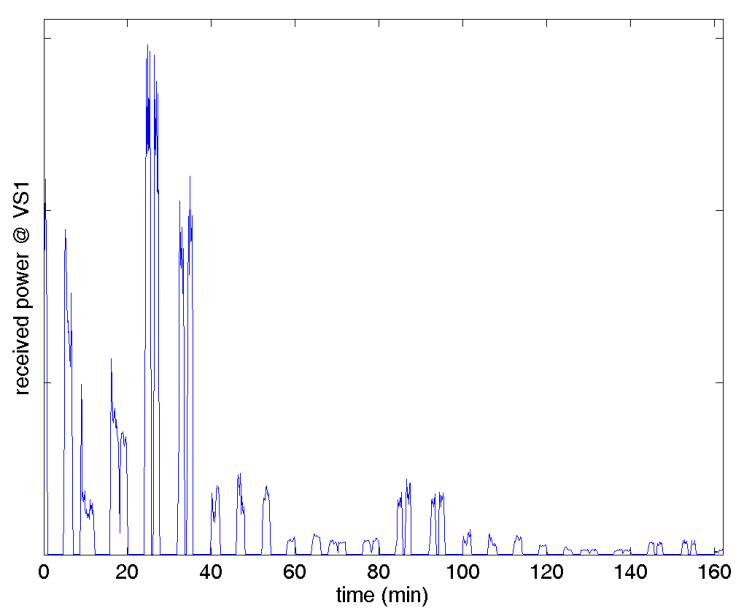

Figure 6. Azimuth estimates of the HF TB2 source during ship's drift, with superimposed source bearing $(\mathrm{X})$ considering ship's heading and estimates in figure 5a) a), signal power received at vector sensor $1 \mathrm{~b}$ ).

alignment. As explained above, the orientation of $x$ and $y$ axis were unknown, thus their orientation was obtained from these ship signature azimuth estimates.

Figure 6a) shows the azimuth estimates for each of the 4 vector sensors, and $b$ ) the average power received by vector sensor 1 in the TB2 source band. It should be remarked that the same estimation procedure were used independently of the type, broadband or narrowband, of the signals.

The time evolution of these single vector sensor estimates, considering the orientation of the vector sensors determined from the azimuth estimates of the ship's signature, are in line with the known geometry of the experiment and comparable with those obtained with the full vector sensor array method described in [11]. The worst results occurs in the initial period, as explained above due to displacements 
of the vector sensors from their vertical alignment. Those perturbations were also noticed in azimuth estimates of ship's noise, however, as expected the estimates obtained with these much higher frequency signals are more sensitive to sensor displacements and the perturbations last longer. Otherwise the estimates obtained from all sensors are in good agrement, with small variances, considering the high frequencies, increasing distance and consequently the lower received power.

\section{CONCLUSION}

This paper discussed time and frequency domain implementations of an intensity based method in order to estimate the azimuth of a source using a single vector sensor. It was shown, with field data, that a vector sensor suspended from a low velocity moving ship is able to track a high frequency bottom moored source and a low frequency ship noise signature, consistently during more than two hours period. The estimates obtained by individual vector sensors are similar to those obtained by full array beamforming for the same data set and acquisition system. Furthermore, the methods are easy to implement with low computational demand, thus suitable to be used in real-time processing in moving or light platforms. A performance and sensitivity study of method should be considered in future work, bearing in mind its usage in mobile/light platforms to $3 \mathrm{D}$ localization and geoacoustic inversion.

\section{ACKNOWLEDGMENT}

The authors would like to thank Michael Porter, chief scientist for the Makai Experiment, Bruce Abraham at Applied Physical Sciences for providing assistance with the data acquisition, the SPAWAR Systems Center in San Diego for providing the source, and the team at HLS Research for their help with the data used in this analysis. The authors also thank Jerry Tarasek at Naval Surface Weapons Center for the use of the vector sensor array. This work was partially supported by the FCT (ISR/IST plurianual funding) through the PIDDAC Program funds and project SENSOCEAN (PTDC/EEA-ELC/104561/2008).

\section{REFERENCES}

[1] M. T. Silvia and R. T. Richards, "A theoretical and experimental investigation of low-frequency acoustic vector sensors," in Proc. of the OCEANS'02 Conf. Biloxi: MTS/IEEE, October 2002.

[2] G. D'Spain, W. S. Hogkiss, and G. L. Edmonds, "Initial analysis of the data from the vertical difar array," in Proc. of the OCEANS'92 Conf. Newport,RI (USA): MTS/IEEE, October 1992.
[3] P. K. Tam and K. T. Wong, "Cramér-rao bounds for direction finding by an acoustic vector sensor under nonideal gainphase responses, noncollocation, or nonorthogonal orientation," IEEE Sensors Journal, vol. 9, no. 8, pp. 969-982, August 2009.

[4] J. C. Shipps and B. M. Abraham, "The use of vector sensors for underwater port and waterway security," in Proc. ISA/IEEE Sensors for Industry Conf., August 2004.

[5] A. Song, M. Badiey, P. Hursky, and A. Abdi, "Time reversal receivers for underwater acoustic communication using vector sensors," in Proc. of the OCEANS'08 Conf. Quebec City, Canada: MTS/IEEE, September 2008.

[6] P. Santos, O. Rodriguez, P. Felisberto, and S. Jesus, "Geoacoustic matched-field inversion using a vertical vector sensor array," in Proc. of 3rd Inter. Conf. and Exhib. on Underwater Acoustic Measurements: Technologies and Results, Nafplion, Greece, 2009.

[7] M. Hawkes and A. Nehorai, "Wideband source localization using a distributed acoustic vector-sensor array," IEEE Trans. Signal Processing, vol. 27, no. 3, pp. 628-637, July 2002.

[8] A. Abdi, H. Guo, and P. Sutthiwan, "A new vector sensor receiver for underwater acoustic communications," in Proc. of the OCEANS'07 Conf. Vancouver, Canada: MTS/IEEE, September 2007.

[9] B. M. Abraham, "Ambient noise measurements with vector acoustic hydrophones," in Proc. of the OCEANS'06 Conf. Bonston (USA): MTS/IEEE, September 2006.

[10] A. Nehorai and E. Paldi, "Acoustic vector-sensor array processing," IEEE Trans. Signal Processing, vol. 42, no. 9, pp. 2481-2491, September 1994.

[11] P. Santos, P. Felisberto, and P. Hursky, "Source localization with vector sensor array during the makai experiment," in Proc. of 3rd Inter. Conf. and Exhib. on Underwater Acoustic Measurements: Technologies and Results, Heraklion, Greece, 2007.

[12] M. Hawkes and A. Nehorai, "Acoustic vector-sensor correlations in ambient noise," IEEE Journal of Oceanic Eng., vol. 26, no. 3, pp. 337-347, July 2001.

[13] A. Abdi and H. Guo, "Signal correlation modeling in acoustic vector sensor arrays," IEEE Trans. Signal Processing, vol. 57, no. 3, pp. 892-903, March 2009. 